\title{
Isolated Tuberculosis of Sacrum with Monoparesis: An Atypical Presentation
}

\author{
Radheshyam Sament, Vikas Bachhal, Nirmal Raj Gopinathan, Ramesh Kumar Sen \\ Department of Orthopaedics, Post Graduate Institute of Medical Education Research, Chandigarh, India
}

\begin{abstract}
Tuberculosis is a major health problem in developing nations. Spine is the most commonly affected site for skeletal tuberculosis but involvement of sacrum is rare. Isolated involvement of sacrum has been reported in literature but none of the reports has mentioned its clinical presentation as monoparesis. Our case presented with symptoms of sensory and motor deficit in right lower limb. The magnetic resonance imaging spine and non contrast computerized tomogram revealed a sacral lesion but were inconclusive of diagnosis. Histological examination after computed tomography guided biopsy revealed the condition as tuberculosis. Anti tubercular treatment was started after confirmation of diagnosis and continued for 18 months. Erythrocyte sedimentation rate and C-reactive protein drooped to normal range and patient was symptom free at two-year follow up. This case report intends to emphasize that sacral tuberculosis, being itself a rare condition, may present atypically as monoparesis.
\end{abstract}

Keywords: Tuberculosis; Sacroiliac joint; Antibiotics; Monoparesis

\section{Introduction}

Musculoskeletal tuberculosis accounts for $3 \%$ of all cases of tuberculosis [1]. Although $50 \%$ of these cases are spinal, infection of the lumbosacral junction with Mycobacterium tuberculosis is quite rare and occurs in only $2 \%$ to $3 \%$ of all cases of spinal tuberculosis [1-3]. Involvement of sacrum is usually secondary to lumbar spine and is mainly caused by direct spread of infection. Only few case reports mentioned in literature were clinically presented as low back pain and treated for a long time with analgesics and rest, before appropriate diagnosis could be made. Isolated involvement of sacrum is very rare. We present here a case of spinal tuberculosis with isolated involvement of sacrum with monoparesis of right lower limb.

\section{Case Report}

A 20-year-old male presented with complaint of weakness in right lower limb and loss of sensation. He was having low back pain for past two months for which he was being treated by analgesics and rest. There was history of low-grade fever, malaise, and significant weight loss in the last 2 months. Neurological examination revealed that findings were localized to the right lower limb. Bulk and tone of right calf were less than the left calf. There were no neurological deficits in the left lower limb. Nevertheless, in the right lower limb, the motor powers were of $4 / 5$ grade around hip, $3 / 5$ at knee joint, and 2/5 at ankle joint. Knee jerk was sluggish and ankle jerk was absent on the right side. Superficial abdominal reflexes were present bilaterally. Plantar reflex was absent on the right side. Pain and temperature sensation was impaired in the

Received Apr 26, 2012; Revised Jul 6, 2012; Accepted Jul 7, 2012

Corresponding author: Radheshyam Sament

Department of Orthopaedics, Post Graduate Institute of Medical Education Research,

Sector 12, Chandigarh, 160012, India

Tel: +91-9660007476, Fax: +91-01412547680, E-mail: samantradhe@gmail.com 
L4, L5, and S1 distribution in right lower limb. Perianal and perineal sensation were present. Urinary bladder was not distended and bowel habits were normal. No gibbus or kyphosis was found on examination of the spine. Straight-leg raising test was positive on the right side. Clinical presentation suggested, lesion of some compressive etiology in region of lumbosacral spine, which was giving lower motor neuron type of clinical picture.

On laboratory investigation, hemoglobin level was 8.7 $\mathrm{g} / \mathrm{dL}$, erythrocyte sedimentation rate (ESR) was $60 \mathrm{~mm} / 1$ hour and; C-reactive protein (CRP) was $40 \mathrm{mg} / \mathrm{L}$; other biochemical parameters were normal. The human immunodeficiency virus (HIV) and enzyme-linked immunosorbent assay were negative. Mantoux test was positive with an induration of $20 \times 15 \mathrm{~mm}$. X-ray of lumbosacral spine lateral and anteroposterior views and $\mathrm{X}$-ray chest posteroanerior view were normal. The non contrast computerized tomogram (NCCT) showed a large lobulated hypodence mass with destruction of right sacral ala and posterior elements with spread of lesion to right piriformis muscle (Fig. 1). The magnetic resonance imaging
(MRI) revealed large mass causing osseous destruction of all sacral vertebral segments and their posterior elements. Mass was extended to presacral area anteriorly and sacral canal posteriorly along with contagious spread to right piriformis muscle. Intervertebral disc of L5-S1 was intact with normal lumbar vertebrae. Lesion showed hyperintence signals in $\mathrm{T} 2 \mathrm{~W}$ images and mild hypointence signals in T1W images (Fig. 2). Finding were suggestive of a mitotic bony lesion; chordoma; Ewing sarcoma; tuberculosis.

Fine needle aspiration cytology (FNAC) from posterior part of mass was performed to define the nature of lesion. Smear showed scattered spindle cells and fibro collagenous tissue. No features of any malignant lesion were observed. Staining of material by zeal-nelson stain was negative for acid fast bacilli (AFB). A computed tomography (CT) guided biopsy was planned to confirm histopathological diagnosis. Biopsy was taken from peripheral margins of lesion under CT guidance. Histopathological examination of tissue showed presence of tuberculous granuloma. Central caseous necrosis was surrounded by
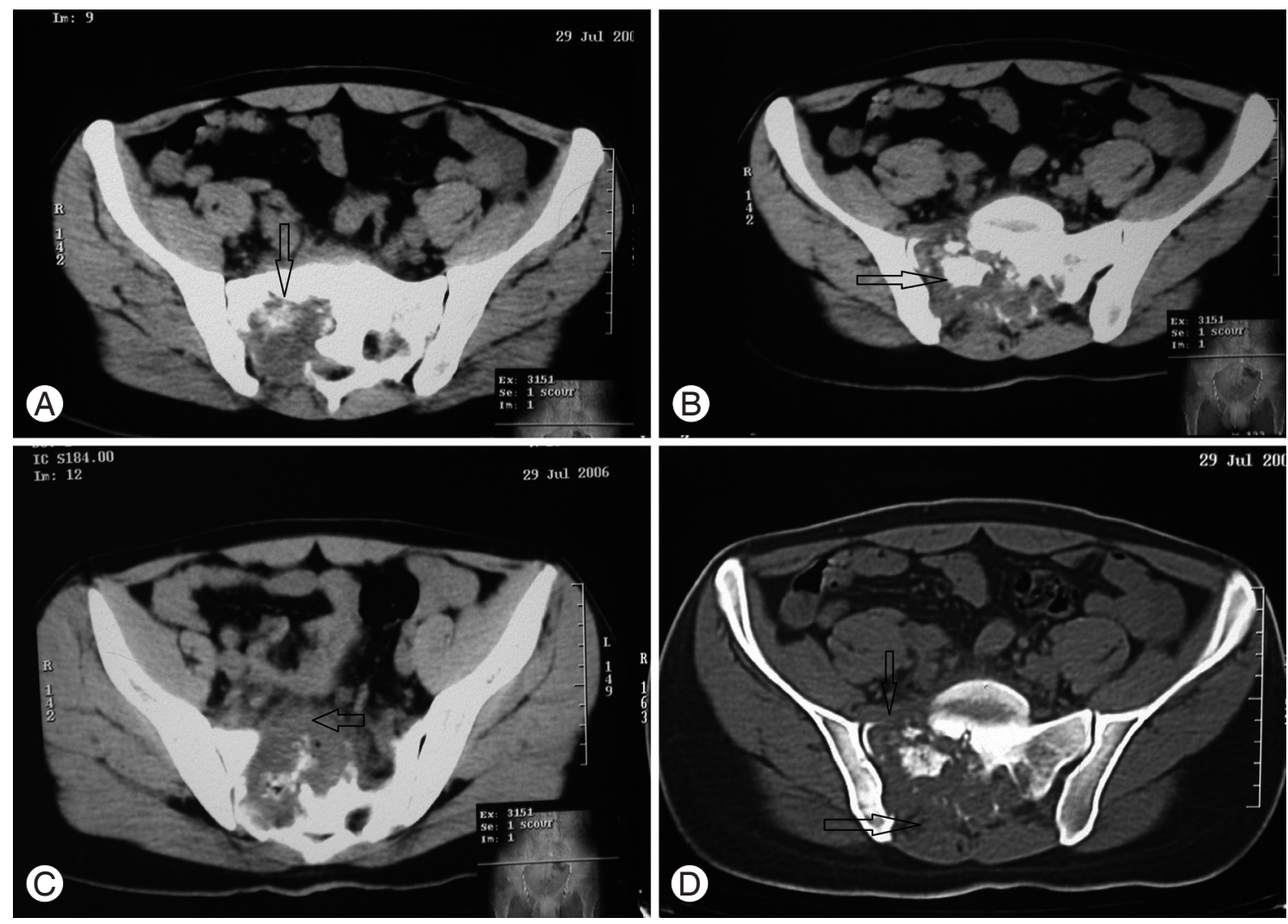

Fig. 1. Non contrast computerized tomogram of sacrum. (A) Shows predominant destruction of right sacral ala. (B) Sequestrum with in caseous necrotic material. (C) Extension of pus anteriorly. (D) Extension of lesion in anterior and posterior elements. 
epithelioid cells and Langhans type giant cells. This finding was consistence with tuberculosis as a cause of bony destruction.

Treatment was initiated with a four-drug antibiotic regimen (isoniazid, rifampicin, pyrazinamide, ethambutol) for initial intensive phase of three month followed by continuation phase with two drug regimen (isoniazid, rifampicin) for next 15 months. Improvement in symptoms and laboratory marker (ESR, CRP) were assessed periodically, at monthly intervals for initial 6 months when value of these specific markers returned to normal levels (ESR $=6$ $\mathrm{mm} / 1 \mathrm{st}$ hour; $\mathrm{CRP}=5 \mathrm{mg} / \mathrm{L}$ ). The patient was found to gradually recovered from monoparesis. At the latest follow up, after 2 years of starting antibiotics, patient was ambulatory with no symptoms of residual disease.

\section{Discussion}

Tuberculosis continues to result in significant morbidity and mortality despite of universal availability of effective multidrug chemotherapy. The emergence of multi-drugresistant mycobacteria coupled with a worldwide increase in HIV infection has led to a recent upsurge in patients with tuberculosis. Tuberculosis remains one of the most pressing health problems in the developing world. Spinal tuberculosis has various atypical features, which include single vertebral infection [4], skip lesions, neural arch involvement and atypical sacral lesions [5]. It usually follows hematogenous spread from the lung or genitourinary tract, with the paravertebral venous plexus of Batson being the likely primary pathway for dissemination of the
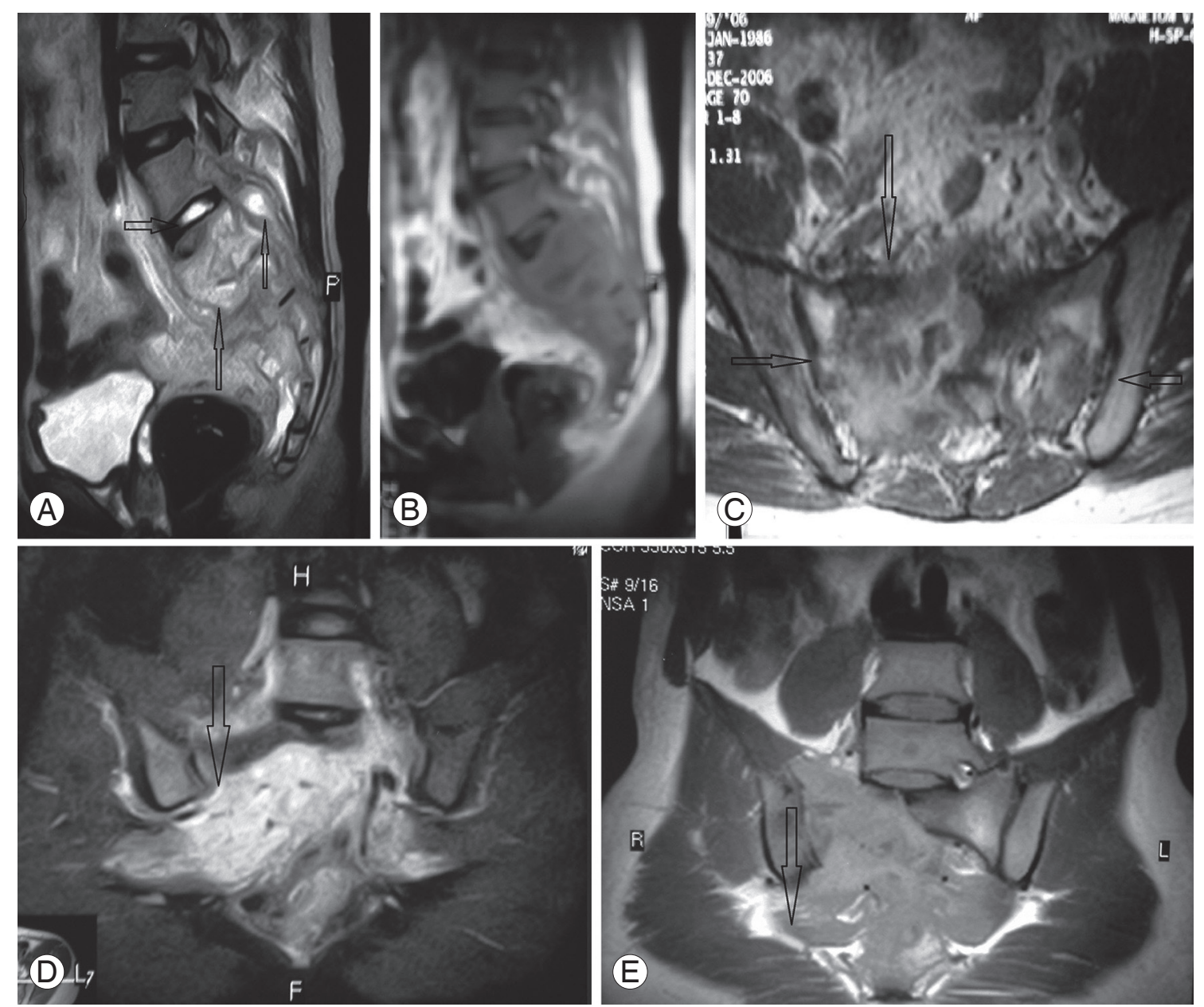

Fig. 2. Magnetic resonance imaging showing (A) sagittal T2W images showing hyperintence signals from lesion; intact L5S1 disc (horizontal arrow) and spread of lesion anteriorly to viscera and posteriorly to sacral canal (vertical arrows). (B) T1W image of the same site. (C) Axial section at the level of sacroiliac (SI) joints demonstrates involvement mainly on right side (vertical arrow) and intact SI Joints (horizontal arrow). (D, E) T1W and T2W image spread of infection along lumbosacral plexus and right piriformis. 
bacilli to the vertebral column [6].

Rajasekaran and Dheenadhyalan [1] reported a 15-year follow-up of 53 patients and compared them for lesions extending to sacrum. Of these only 12 had spread disease inferiorly to lumbosacral junction. Pun et al. [7] reported 20-year follow-up of 26 patients of tuberculosis of lumbosacral junction. In majority of the patients, the disease had started in lumbar spine and descended to the sacrum. Dayras et al. [8] reported first case of isolated sacral tuberculosis with lower back pain. In 2004, Wellons et al. [9] presented a case of sacral tuberculosis with lower back pain and difficulty in walking with bilateral involvement of lower limbs. Punia and Kumar [10] showed that sacral tuberculosis could present as quada-conus syndrome.

Isolated sacral tuberculosis is a very rare condition, and to the best of our knowledge, its presentation as monoparesis has not been reported in literature so far. Our case presented with features of monoparesis in right lower limb. This might have resulted from involvement of right lumbosacral plexus. A case of sacral tuberculosis presenting with such presentation is not only atypical, but also it can cause difficulty and delay in making a diagnosis. Plain radiographs are extremely insensitive; they do not detect vertebral involvement until at-least 50\% of a vertebra is destroyed [7]. We encountered similar problem, as X-ray findings were not suggestive of any lesion. Even investigations including MRI and NCCT were inconclusive and were more suggestive of some kind of malignant bone lesion. Furthermore, FNAC did not show any evidence of $\mathrm{AFB}$, thus making diagnosis more obscured. Only positive findings towards tuberculosis were high ESR levels and positive Mantoux test. Both of these were neither sensitive nor specific enough to confirm the diagnosis. Based on the present case, it is proposed that if we find the tuberculous granuloma with epithelioid cells and Langhans giant cells surrounding central area in the histopathological examination of tissue obtained by CT guided biopsy, the tuberculosis should be diagnosed finally. Tuberculosis of bones has been known to present itself in a variety of atypical clinical forms. This interesting case of isolated sacral tuberculosis with sensory and motor deficit was unique and emphasizes importance of atypical presentation of bony tuberculosis.

\section{Conflict of Interest}

No potential conflict of interest relevant to this article was reported.

\section{References}

1. Rajasekaran S, Dheenadhyalan J. Tuberculosis of bone and joints. In: Bulstrode C, Buckwalter J, Carr A, et al., editors. Oxford textbook of orthopaedics and trauma. Oxford: Oxford University Press; 2002. p.1532-60.

2. Tuli SM. Clinical features. In: Tuli SM, editor. Tuberculosis of skeletal system: bones, joints, spine and bursal sheaths. 2nd ed. New Delhi: Jaypee Brothers; 1997. p.177-82.

3. Seidel K. Anal fistula cause by sacrococcygeal tuberculosis. Z Tuberk 1950;94:307-9.

4. Naim-Ur-Rahman, El-Bakry A, Jamjoom A, Jamjoom ZA, Kolawole TM. Atypical forms of spinal tuberculosis: case report and review of the literature. Surg Neurol 1999;51:602-7.

5. Patankar T, Krishnan A, Patkar D, et al. Imaging in isolated sacral tuberculosis: a review of 15 cases. Skeletal Radiol 2000;29:392-6.

6. Ahmadi J, Bajaj A, Destian S, Segall HD, Zee CS. Spinal tuberculosis: atypical observations at MR imaging. Radiology 1993;189:489-93.

7. Pun WK, Chow SP, Luk KD, Cheng CL, Hsu LC, Leong JC. Tuberculosis of the lumbosacral junction: long-term follow-up of 26 cases. J Bone Joint Surg Br 1990;72:675-8.

8. Dayras JC, Lorilloux J, Hugonet M, Benichou P. Osseous tuberculosis of the sacrum. Ann Pediatr (Paris) 1985;32:289-93.

9. Wellons JC, Zomorodi AR, Villaviciencio AT, Woods CW, Lawson WT, Eastwood JD. Sacral tuberculosis: a case report and review of the literature. Surg Neurol 2004;61:136-9.

10. Punia VP, Kumar S. Atypical manifestation of sacral tuberculosis as cauda conus syndrome. J Indian Acad Clin Med 2008;9:57-60. 